\title{
Magnetic diffusivities in 3D radiative chemo-hydrodynamic simulations of protostellar collapse
}

\author{
Natalia Dzyurkevich ${ }^{1}$, Benoît Commerçon ${ }^{2}$, Pierre Lesaffre ${ }^{1}$, and Dimitry Semenov ${ }^{3}$ \\ ${ }^{1}$ Laboratoire de Radioastronomie Millimétrique, UMR 8112 du CNRS, École Normale Supérieure et Observatoire de Paris, \\ 24 rue Lhomond, 75231 Paris Cedex 05, France \\ e-mail: natalia@lra.ens.fr \\ 2 École Normale Supérieure de Lyon, CRAL, UMR 5574 du CNRS, Université de Lyon I, 46 allée d'Italie, 69364 Lyon Cedex 07, \\ France \\ 3 Max-Planck-Institut für Astronomie, Königstuhl 17, 69117 Heidelberg, Germany
}

Received 25 May 2016 / Accepted 12 March 2017

\begin{abstract}
Context. Both theory and observations of star-forming clouds require simulations that combine the co-evolving chemistry, magnetohydrodynamics, and radiative transfer in protostellar collapse simulation. A detailed knowledge of self-consistent chemical evolution for the main charge carriers (both gas species and dust grains) allows us to correctly estimate the rate and nature of magnetic dissipation in the collapsing core. This knowledge is critical to answer one of the most significant issues of star and planet formation: what is the magnitude and spatial distribution of magnetic flux as the initial condition to protoplanetary disk evolution?

Aims. We use a chemo-dynamical version of RAMSES, which is described in a companion publication, to follow the chemo-dynamical evolution of collapsing dense cores with various dust properties and interpret differences that occur in magnetic diffusivity terms. These differences are crucial to circumstellar disk formation.

Methods. We performed 3D chemo-dynamical simulations of $1 M_{\odot}$ isolated dense core collapse for a range in dust size assumptions. The number density of dust and its mean size affect the efficiency of charge capturing and the formation of ices. The radiative hydrodynamics and dynamical evolution of chemical abundances were used to reconstruct the magnetic diffusivity terms for clouds with various magnetisation.

Results. The simulations are performed for a mean dust size ranging from $0.017 \mu \mathrm{m}$ to $1 \mu \mathrm{m}$, and we adopt both a fixed dust size and a dust size distribution. The chemical abundances for this range of dust sizes are produced by RAMSES and serve as inputs to calculations of Ohmic, ambipolar, and Hall diffusivity terms. Ohmic resistivity only plays a role at the late stage of the collapse in the innermost region of the cloud where gas density is in excess of a few times $10^{13} \mathrm{~cm}^{-3}$. Ambipolar diffusion is a dominant magnetic diffusivity term in cases where mean dust size is a typical ISM value or larger. We demonstrate that the assumption of a fixed dominant ion mass can lead to a one order of magnitude mismatch in the ambipolar diffusion magnitude. The negative Hall effect is dominant during the collapse in case of small dust, i.e. for the mean dust size of $0.02 \mu \mathrm{m}$ and smaller; we connect this effect to the dominance of negatively charged grains. We find that the Hall effect reverses its sign for mean dust size of $0.1 \mu \mathrm{m}$ and smaller. The phenomenon of the sign reversal strongly depends on the number of negatively charged dust relative to the ions and the quality of coupling of the charged dust to the magnetic fields. We have adopted different strengths of magnetic fields, $\beta=P_{\text {gas }} / P_{\text {mag }}=2,5$, 25. We observe that the variation on the field strength only shifts the Hall effect reversal along the radius of the collapsing cloud, but does not prevent it.

Conclusions. The dust grain mean size appears to be the parameter with the strongest impact on the magnitude of the magnetic diffusivity, dividing the collapsing clouds in Hall-dominated and ambipolar-dominated clouds and affecting the size of the resulting disks. We propose to link the dust properties and occurrence and size of disk structures in Class 0 young stellar objects. The proper accounting for dust grain growth in the radiative magneto-hydrodynamical collapse models are as important as coupling the dynamics of the collapse with the chemistry.
\end{abstract}

Key words. magnetohydrodynamics (MHD) - ISM: clouds - astrochemistry - diffusion

\section{Introduction}

The Ophiuchus, Serpent, and Taurus-Orion clouds are rich in young stellar objects (YSOs) with the total number is close to five thousand. Nevertheless, Class 0 YSO are rare owing to the relatively short time window in which collapse of the cloud occurs. Additionally, those objects are distant and can be 130 to 500 parsecs away. This and the fact that they are heavily veiled by the envelope makes the observations of their internal structure quite challenging. Nevertheless, there are at least four known Class 0 objects possessing large ( $>100 \mathrm{AU})$ rotationally supported disks, i.e. L 1527, VLA 1623A, RCrA IRS7B, and HH212 MMS Tobin et al. (2015). More candidates have been added to this list in the last year (Tobin et al. 2016b), however difficulties in recovering the rotation in the disks via molecular lines remain. The dense compact structures detected in most of the Class 0 objects are small (less than $100 \mathrm{AU}$ ) and remain unresolved. The new powerful instruments allow us to study the close-by objects in more detail; these include the gravitational disk fragmentation resulting in the triple binary in L1448 IRS3B (Tobin et al. 2016a), the disk-envelope study made with ALMA for VLA 1623 (Murillo et al. 2015), and the spiral arms in the young disk in Elias 2-27 (there are also disagreements about its age; Pérez et al. 2016). Those observations indicate that there are more small disk cases versus more rare prominent large disks around YSO Class 0. 
It is interesting to compare those trends to the results of the numerical simulations. Magnetic diffusivity terms are proposed as a way out of the magnetic braking catastrophe observed in ideal magneto-hydrodynamical (MHD) simulations of the collapse (e.g. Hennebelle \& Fromang 2008; Commerçon et al. 2010). Within the ideal MHD approach, the misalignment of rotation axis and magnetic field (Joos et al. 2012), turbulence (Joos et al. 2013; Seifried et al. 2015), and reconnection processes (Lazarian et al. 2012) can help to remove the magnetic flux. In addition, the non-ideal MHD effects, including Ohmic diffusion, ambipolar diffusion, and the Hall effect, are natural consequences of gas conditions in dense molecular clouds and happen to reduce the magnetic braking efficiency, allowing for the disk to form (e.g. Li et al. 2011; Dapp et al. 2012; Tomida et al. 2015; Masson et al. 2016; Tsukamoto et al. 2015a; Wurster et al. 2016).

In the last $20 \mathrm{yr}$, a large number of studies were performed modelling the protostellar collapse and disk formation with nonideal MHD with a relatively simple fitting formulae for ion and electron densities. Krasnopolsky et al. (2010) estimated the necessary Ohmic resistivity magnitude to be $10^{19} \mathrm{~cm}^{2} \mathrm{~s}^{-1}$ in order to form a disk of radius $100 \mathrm{AU}$, and these authors also showed that Ohmic resistivity is likely to be much smaller. Indeed, following Nakano et al. (2002), Machida et al. (2007), Tomida et al. (2015) showed that Ohmic resistivity becomes important deep in the cloud within the first hydro-static core (FHSC) when mean dust size is assumed to be rather tiny.

Ambipolar diffusion is also shown to play an important role during cloud collapse and disk formation. Its effect to redistribute the magnetic flux is strongest for low gas densities, where the magnetic field is coupled mostly to the ions and can "slip" through the neutral gas. The variety of studies range from theoretical and semi-analytical models with 1D thin-disk approximation (Mestel \& Spitzer 1956; Basu \& Ciolek 2004; Krasnopolsky \& Königl 2002) to 3D AMR simulations of the cloud collapse with chemical look-up tables as in Masson et al. (2016), Hennebelle et al. (2016). Duffin \& Pudritz (2008, 2009) considered the impact of ambipolar diffusion for dust-free gas in a one-fluid approach. Following Mellon \& Li (2009), they found that the ambipolar diffusion does not weaken the magnetic breaking enough to allow the rotationally supported disk to appear. Their findings were supported by Li et al. (2011), who computed the ion density self-consistently from the Nakano chemical network (Nakano et al. 2002). Tomida et al. (2015) performed 3D nested-grid RMHD simulations of the collapsing cloud and found that only a tiny disk, of less then $5 \mathrm{AU}$ in radius, can be formed around a protostar during the first core collapse. The simulations of Masson et al. (2016) and Hennebelle et al. (2016) show that the formation of the Keplerian disk of $18 \mathrm{AU}$ around the first core is a robust result of self-regulating nature of ambipolar diffusion in the collapsing cloud. In those models they include ambipolar diffusion and Ohmic dissipation, which are calculated with the method shown in Marchand et al. (2016). The similarities and disagreements between the latest simulations are discussed in Masson et al. (2016), where disagreements seem to be caused by the different input parameters for chemical modelling of the gas.

Although the Hall effect is less explored than ambipolar diffusion, this effect is currently the subject of intense investigations ( $\mathrm{Li}$ et al. 2011; Braiding \& Wardle 2012; Tsukamoto et al. 2015a; Wurster et al. 2016). According to Li et al. (2011), the Hall effect is dynamically significant but not capable of forming the rotationally supported disk. In contrast, the results of Tsukamoto et al. (2015b) show that there is a possibility of forming large disks under specific conditions. Its effect depends on the orientation between the rotation axis and magnetic field (parallel or anti-parallel), which results in bimodality in the disk properties (Tsukamoto et al. 2015a). Altogether, it is obvious that magnetic diffusivities need to be taken into account all the way along the collapse to resolve the riddle of protostellar disks formation in the presence of the magnetic field. A detailed description of the dust-gas chemistry, an accurate treatment of processes responsible for the ionisation, and a good model for dust growth are thus highly desirable.

Chemistry is expected to play a crucial role during the star formation process. There are several reasons for making an effort to include the dynamical chemical evolution of key species into the radiative non-ideal MHD simulations of the molecular cloud collapse. Besides being a straight link to observations, chemical abundances can be used to provide additional cooling of gas via atomic and molecular emission lines. The next very important aspect is that chemistry provides a realistic degree of ionisation, ions distribution, and grain charging. As pointed out in $\mathrm{Li}$ et al. (2014) and Inutsuka (2012), we need to consider all three types of magnetic diffusivity (Ohm, ambipolar and Hall) to resolve the long-standing problems of angular momentum and magnetic flux removal during star formation. The accurate calculation of the magnetic dissipations requires a precise description of the charge carriers, which requires the inclusion of chemistry in the calculations. While the cooling with molecular lines is expected to be important in the outer region of the envelope, the impact of chemistry on the magnetic diffusivities is going to be important everywhere, at any radius of the collapsing molecular cloud.

The equilibrium chemical models have been widely used to determine the degree of ionisation (e.g. Nakano et al. 2002; Marchand et al. 2016). These authors deal mostly with representative ions (metals and non-metals), dust, and electrons. Dust is important in the chemical models not only for forming complex molecules on its surface, but also for capturing electrons and neutralising through collisions with ions and charged dust, whereby the detailed physical properties of dust become very important (Marchand et al. 2016). Nakano et al. (2002) assumed a dust size distribution dominated by tiny (a few nm) dust grains, which somewhat contradicts the observations of much larger grains in the dense cloud core, i.e. the so-called "coreshine" (Steinacker et al. 2010). The only known objects without observable coreshine, i.e. with no indications for large grains, are in the Gum/Vela region, where Pagani et al. (2012) suspect a nearby supernova is responsible for large dust destruction.

We merged the RAMSES code (Teyssier 2002) and the thermo-chemical Paris-Durham shock PDS code (Flower \& Pineau des Forêts 2003, 2015; Lesaffre et al. 2005, 2013) with the technical description published in Dzyurkevich et al. (2017, Paper I). Paper I contains the detailed description of the chemical reactions and implementation details, which are also relevant for the simulations presented below. In this paper we ask the question about the coupling of the charged gas-dust mixture with magnetic fields, for various dust sizes. We present the analysis of the magnetic diffusivity terms resulting from the dynamical chemical evolution for the radiation-(M)HD models of protostellar collapse. There have been a few publications that can be considered precursors to our study. Tassis et al. (2012a,b,c) applied a complex chemical network to the isothermal phase of typical prestellar cloud collapse, performing a wide parameter study and searching for related pairs of neutral-ion molecules to provide sensitive probes of the importance of magnetic fields and ambipolar diffusion in such clouds. We considered the pairs of $\mathrm{HCO}^{+}, \mathrm{CO}$ and $\mathrm{H}_{3} \mathrm{O}^{+}, \mathrm{H}_{2} \mathrm{O}$ in the companion publication 
Paper I. In spite of increased computational costs, the models such as presented in Paper I are self-consistent and can be used threefold: for matching the observations, for calculating non-ideal MHD terms, and for thermal chemical feedback (i.e. cooling with atomic or molecular lines) (Hincelin et al. 2016; Harsono et al. 2015; Gerin et al. 2015). We leave the cooling of atomic and molecular lines for follow-up studies. The goal of this paper is to demonstrate the severity of the dust properties for magnetic dissipation during the collapse. This is an important piece of information allowing us to digest the previously performed collapse simulations with Hall and ambipolar diffusivities, and to make a theoretical prediction for the initial conditions for protoplanetary disks.

The paper is organised as follows. In Sect. 2, we present the terse description of the reduced chemical network we use for protostellar collapse and the coupling of chemistry and radiation-hydrodynamics in RAMSES, whereas the details can be recovered in Paper I. We present the set-up for the collapse simulation and analyse the magnetic diffusivities for fiducial model in Sect. 3. We discuss the effects of the various dust sizes on the non-ideal MHD terms in Sect. 4. Section 5 concludes our work.

\section{Dynamical chemistry in RAMSES}

In this section we briefly introduce the chemo-dynamical modification of RAMSES (Teyssier 2002), which we described in detail in Paper I. We first describe the reduced chemical network that we use for protostellar applications, and then we describe the chemical equations in the context of RAMSES.

\subsection{Reduced chemical network}

The chemical network we designed represents the main species and reactions necessary to describe $\mathrm{CO}$ abundances within mainly H-C-O chemistry in the early phases of protostellar collapse (Lesaffre et al. 2005). We include 14 neutral species $(\mathrm{H}$, $\mathrm{H}_{2}, \mathrm{He}, \mathrm{C}, \mathrm{CH}_{x}$ with $x=1, \ldots, 4, \mathrm{O}, \mathrm{O}_{2}, \mathrm{H}_{2} \mathrm{O}, \mathrm{OH}, \mathrm{CO}$, and $\mathrm{CO}_{2}$ ), their corresponding single-charged positive ions and ionised molecules $\mathrm{CH}_{5}^{+}, \mathrm{H}_{3} \mathrm{O}^{+}, \mathrm{HCO}^{+}$, and $\mathrm{H}_{3}^{+}$. In addition, iron is also included as a representative metal. For the details on the total elemental abundance or the dust elemental composition, please consult Paper I. We constrain ourselves here to the description of the dust properties.

The size and mass of dust grains are calculated from the total mass of "dust-core" and "dust-mantle" elements and from the adopted size distribution. The number of dust grains are calculated as $n_{\text {dust }}=M_{\mathrm{G} \text {,total }} /\left((4 / 3) \pi \rho_{\text {solid }}\langle a\rangle^{3}\right)$, where $\rho_{\text {solid }}$ is the internal density, $M_{\mathrm{G} \text {,total }}$ is the total mass of the dust core elements, and $\langle a\rangle$ is the mean radius calculated via the MRN size distribution $n(a) \propto a^{-3.5}$ (Mathis et al. 1977, see Appendix A). All dust core species do not participate in the chemical reactions but are used exclusively to calculate dust grain mass.

All neutral species are allowed to freeze out on the dust grains, thus forming the grain mantles and increasing the weight of the grains. Last, we consider three type of grains, namely neutral $(\mathrm{G})$, single-charged positive $\left(\mathrm{G}^{+}\right)$, and single-charged negative $\left(\mathrm{G}^{-}\right)$. In total, the reduced chemical network describes the evolution of $N_{\text {species }}=56$ species.

We include gas-phase, freeze out, and sublimation chemical reactions. We do not take into account other types of reactions such as soft X-ray ionisation, which is important for low density (visual extinction $A_{\mathrm{v}} \sim 0.2$; Wolfire et al. 1995). For protostellar collapse applications, $A_{\mathrm{v}}$ is already larger than 10 for a typical mass of $1 M_{\odot}$.
In total, our reduced gas-grain chemical network for $\mathrm{H}-\mathrm{C}-\mathrm{O}$ (and $\mathrm{Fe}$ as a representative metal) includes 231 reactions.

\subsection{Chemo-radiation-hydrodynamic model}

We performed 3D time-dependent chemical calculations of protostellar collapse using the time-dependent chemistry code PDS code (Flower \& Pineau des Forêts 2015, 2003; Lesaffre et al. 2005, 2013) coupled with the RAMSES code (Teyssier 2002). For the technical details, we refer to Paper I. Below we provide a brief summary of the code characteristics.

The set of chemo-RHD equations solved in RAMSES with all radiative quantities estimated in the co-moving frame are

$$
\begin{cases}\partial_{t} \rho+\nabla \cdot[\rho \boldsymbol{u}] & =0 \\ \partial_{t} \rho \boldsymbol{u}+\nabla \cdot[\rho \boldsymbol{u} \otimes \boldsymbol{u}+P \mathbb{I}]= & -\lambda \nabla E_{\mathrm{r}} \\ \partial_{t} E_{\mathrm{T}}+\nabla \cdot\left[\boldsymbol{u}\left(E_{\mathrm{T}}+P\right)\right]= & -\mathbb{P}_{\mathrm{r}} \nabla \cdot \boldsymbol{u}-\lambda \boldsymbol{u} \nabla E_{\mathrm{r}} \\ & +\nabla \cdot\left(\frac{c \lambda}{\rho \kappa_{\mathrm{R}}} \nabla E_{\mathrm{r}}\right) \\ \partial_{t} E_{\mathrm{r}}+\nabla \cdot\left[\boldsymbol{u} E_{\mathrm{r}}\right] & -\mathbb{P}_{\mathrm{r}} \nabla \cdot \boldsymbol{u}+\nabla \cdot\left(\frac{c \lambda}{\rho \kappa_{\mathrm{R}}} \nabla E_{\mathrm{r}}\right) \\ & +\kappa_{\mathrm{P}} \rho c\left(a_{\mathrm{R}} T^{4}-E_{\mathrm{r}}\right) \\ = & \sum_{p, q} k_{x p q} n_{p} n_{q}+\sum_{p} l_{x p}^{\mathrm{EP}} n_{p} \\ & -\sum_{x} l_{x}^{\mathrm{FRZ}} n_{x}+\sum_{x} l_{x}^{\mathrm{DES}} n_{x},\end{cases}
$$

where $\rho$ is the material density, $\boldsymbol{u}$ the velocity, $P$ the thermal pressure, $\kappa_{\mathrm{R}}$ the Rosseland mean opacity, $\lambda$ the radiative flux limiter (e.g. Minerbo 1978), $E_{\mathrm{T}}$ the total energy $E_{\mathrm{T}}=\rho \epsilon+1 / 2 \rho u^{2}+E_{\mathrm{r}}$ ( $\epsilon$ is the internal specific energy), $\kappa_{\mathrm{P}}$ the Planck opacity, $E_{\mathrm{r}}$ the radiative energy, and $\mathbb{P}_{\mathrm{r}}$ the radiation pressure.

We note that compared to classical RHD solvers, we now have additional equations on the number density of each species $n_{x}$, which correspond to the advection, chemical creation, and destruction of the $x$ chemical elements. The dust grains, whether charged or not, are treated in exactly same way as the chemical species; they are advected with the gas velocities. We consider here neither a possibility of dust having a different velocity nor the dust enrichment within the cloud (Bellan 2008). On the right side, the four terms represent creation or destruction of specie $x$. First term is describing the two-body Arrhenius-type reactions, second term is for ionization and dissociation by external particles or photons (EP), third and forth terms are due to freeze-out (FRZ) and desorption (DES) of the $x$-species on the dust surface. $k_{x p q}, l_{x p}^{\mathrm{EP}}, l_{x}^{\mathrm{FRZ}}$ and $l_{x}^{\mathrm{DES}}$ are the corresponding reaction rates. The cooling via molecular and atomic lines is present in the PDS chemical code. In the context of this paper, we neglect the thermal feedback of chemistry on the gas to concentrate on which parameters are affecting the abundances of the collant.

We use the time-dependent chemistry PDS code as a module incorporated in RAMSES code. PDS code was originally written for MHD shocks (Flower \& Pineau des Forêts 2015, 2003; Lesaffre et al. 2004, 2005, 2013). We solve the right side of the system of equations for chemical evolution of species $n_{x}$ (see last line in Eq. (1)) using either explicit or implicit integration in time. When the chemical time step is shorter than the hydrodynamical time step, we use an implicit solver that consists in inverting the matrix of $N_{\text {species }}^{2}$ in size for a hydro(-magnetic) time-step of $\Delta t_{\text {hydro. }}$ Should this not be the case, the chemical module switches to an explicit second-order Runge-Kutta method for time advancement. In the implicit scheme, we use 
the DVODE solver ${ }^{1}$ without any optimisation of the matrix inversion procedure.

The methods in AMR RMHD code RAMSES used to solve the problem of collapse are described below. For the radiation transfer, we use the grey flux-limited diffusion (FLD) approximation described in detail in Commerçon et al. (2011), which combines the explicit second-order Godunov solver of RAMSES for the hydrodynamical part, and an implicit scheme for radiative energy diffusion and coupling between matter and radiation terms. The implicit FLD solver uses adaptive time stepping (ATS; Commerçon et al. 2014).

The MHD part of the code is based on the constrained transport (CT) scheme (Teyssier et al. 2006), using a 2DRiemann solver to compute the electro-motive force at cell edges (Fromang et al. 2006; Teyssier et al. 2006). In addition to the ideal MHD solver, ambipolar diffusion and Ohmic diffusion are also implemented as additional electro-motive forces in the induction equation (Masson et al. 2012). The aim of this paper is to estimate the relative importance of various magnetic diffusivity terms, depending on the fixed magnetic field strength, progress of the collapse and dust properties.

The model of chemo-dynamical collapse presented here has its limitations. The PDS chemical code has no impact on the evolution of the gas in RHD part of RAMSES. The possible limitations are then threefold. First, the total gas and dust density $\rho$ are not recomputed from the abundances obtained by the chemistry solver. In this paper, we consider FHSC before the dust melting occurs above $800 \mathrm{~K}$. Second, as mentioned previously, we do not include heating and cooling because of atomic and molecular lines, which should not be dominant as long as radiative transfer is dominated by the dust. Last, we do not self-consistently compute the dust opacity from the dust composition given in output by the chemistry solver. For the latter, we instead use tabulated opacities from Semenov et al. (2003). All these limitations could be dealt by the two codes, but go far beyond the scope of the paper.

\section{Dense core collapse calculations: set-up}

\subsection{Initial conditions}

Parameters of the molecular cloud are listed below. We choose a spherically symmetric collapse configuration, i.e. we neglect rotation, magnetic fields, and turbulence. As we mentioned before, the aim of this paper is to demonstrate the impact of dust properties on the magnetic diffusivities during the collapse. We make use of spherical symmetry and present easy-to-read 1D interpretations of the results. The initial core mass is fixed to $1 M_{\odot}$ and the temperature of both gas and dust is uniform and equals $10 \mathrm{~K}$. In our model, we assume that the dust and gas are perfectly coupled thermally. The adiabatic index is $\gamma=5 / 3$ and the mean molecular weight is $\mu_{\text {gas }}=2.375$. In this paper we use both $n_{\text {gas }}=\rho_{\text {gas }} /\left(\mu_{\text {gas }} m_{\mathrm{p}}\right)$ and $n[\mathrm{H}]=n_{\text {gas }} / \mu_{\text {gas }}$, where $m_{\mathrm{p}}$ is the mass of proton. We represent the relative abundance of $x$ chemical species as $n[x] / n[\mathrm{H}]$

In all our models, the initial density profile is Bonnor-Ebert, similar to $n=n_{\mathrm{c}} /\left(1+\left(r / r_{\mathrm{c}}\right)\right)^{-2}$, where the maximum density in the centre $n_{\mathrm{c}}$ is 10 times larger than the density at the border of the core. The total length of the simulation box is four times larger than the core initial radius $r_{0}$. The central density is $n_{\mathrm{c}}=$ $4.4 \times 10^{5} \mathrm{~cm}^{-3}$ (or, $1.71 \times 10^{-18} \mathrm{~g} \mathrm{~cm}^{-3}$ ) and the core radius is

\footnotetext{
1 From the ODEPACK package downloadable at https:
} //computation.1lnl.gov/casc/odepack/
Table 1. 3D chemo-dynamical calculations of collapse.

\begin{tabular}{llll}
\hline \hline Model & $\begin{array}{l}\sqrt{\left\langle a^{2}\right\rangle} \\
{[\mu \mathrm{m}]}\end{array}$ & $n_{\text {dust }} / n[\mathrm{H}]$ & $\begin{array}{l}\left\langle a^{2}\right\rangle n_{\text {dust }} / n[\mathrm{H}] \\
{\left[(\mu \mathrm{m})^{2}\right]}\end{array}$ \\
\hline $\mathrm{S} 1$ & 0.1 & $3.1 \times 10^{-12}$ & $3.1 \times 10^{-14}$ \\
$\mathrm{~S} 2_{\text {MRN }}$ & 0.05 & $3.9 \times 10^{-12}$ & $1.0 \times 10^{-14}$ \\
$\mathrm{~S} 3_{\text {MRN }}$ & 0.017 & $5.2 \times 10^{-11}$ & $1.5 \times 10^{-14}$ \\
S4 & 1.00 & $3.1 \times 10^{-15}$ & $3.1 \times 10^{-15}$ \\
S5 & 0.02 & $3.9 \times 10^{-10}$ & $1.4 \times 10^{-13}$ \\
\hline
\end{tabular}

Notes. The cloud is assumed to be always of one solar mass. S1 is a fiducial model. S[2, 3] $]_{\mathrm{MRN}}$ are the models with MRN dust size distribution, whereas models $\mathrm{S}[1,4,5]$ have fixed grain size. The value $n_{\text {dust }}\left\langle a^{2}\right\rangle$ represents mean dust cross section.

$r_{0}=0.022 \mathrm{pc}$. The relation between thermal and gravitational energies is $E_{\text {th }} / E_{\text {grav }}=0.447$.

Assuming the same core initial properties as model S1, we vary the dust properties for the models $\mathrm{S} 2_{\mathrm{MRN}}$ to $\mathrm{S} 5$.

We generate the initial chemical abundances as following. We adopt a uniform high visual extinction $A_{\mathrm{v}}=30$ and a uniform, cosmic ray ionisation rate of $1.3 \times 10^{-17} \mathrm{~s}^{-1}$. In order to generate the initial chemical abundances, we start from elemental abundances (see Paper I) and let the chemistry evolve for $6 \times 10^{5} \mathrm{yr}$ in the static Bonner-Ebert sphere configuration (e.g. Hincelin et al. 2013). As mentioned previously, the assumptions about dust size distribution differs depending on the model (see Table 1)

As already introduced in Paper I, we define zero-time $t_{0}$ as the moment of the FHSC formation, i.e. when central density reaches $10^{13} \mathrm{~cm}^{-3}(T \sim 210 \mathrm{~K})$. The zero-time can appear different for the collapse simulations of various spatial extend (see Table 1 in Paper I). In the present paper, the time pace of the collapse is identical for all models, so that FHSC is formed at $t_{0}=57.8 \mathrm{kyr}$ after the beginning of the simulation.

\subsection{Dust properties}

We compare five models with different dust size properties: models $\mathrm{S} 1, \mathrm{~S} 2_{\mathrm{MRN}}, \mathrm{S} 3_{\mathrm{MRN}}, \mathrm{S} 4$, and $\mathrm{S} 5$ (Table 1). The $\mathrm{S} 2_{\mathrm{MRN}}$ and $\mathrm{S} 33_{\text {MRN }}$ models make use of the MRN distribution for the grain sizes in the chemistry module. Models S4 and S5 have a fixed grain size (as in fiducial model S1) and serve as boundary cases, where we assume very large and tiny dust. All five models use identical initial conditions and dust-to-gas ratio $f_{\mathrm{dg}}=0.01$, except for the dust size properties.

We refer to Appendix A for a more complete description of the treatment of dust size. In the following, we briefly summarise the differences between these five models:

S1: fiducial case has single-sized dust with $a_{0}=0.1 \mu \mathrm{m}$, dustto-gas ratio, and dust density $n_{\text {dust }}=3.09 \times 10^{-12} n_{\mathrm{H}_{2}}$;

S2 $2_{\text {MRN }}$ : we use the classical MRN dust grain size distribution $n_{\text {dust }}(a) \sim a^{-3.5}$ (Mathis et al. 1977), scaled to match the results of coreshine modelling (Steinacker et al. 2010). The mean dust radius is $\sqrt{\left\langle a^{2}\right\rangle}=0.05 \mu \mathrm{m}$, which corresponds to $a_{\min }=2.6 \times 10^{-6} \mathrm{~cm}$ and $a_{\max }=5 \times 10^{-5} \mathrm{~cm}$. Fixing $f_{\mathrm{dg}}=0.01$, the dust number density is calculated to be $n_{\text {dust }}=3.9 \times 10^{-12} n_{\mathrm{H}_{2}}$ (see Appendix A for details);

$\mathbf{S 3}_{\mathrm{MRN}}$ : same as $\mathrm{S} 2_{\mathrm{MRN}}$, but we adopt $a_{\mathrm{min}}=10^{-6} \mathrm{~cm}$ and $a_{\max }=3 \times 10^{-5} \mathrm{~cm}$ (Flower \& Pineau des Forêts 2003). The mean dust radius is $\sqrt{\left\langle a^{2}\right\rangle}=0.017 \mu \mathrm{m}$ and $n_{\text {dust }}=$ $5.24 \times 10^{-11} n_{\mathrm{H}_{2}}$. This size range resembles the fit for mixed 
composition "large" grains, made of bulk carbonaceous material, close to the range considered in (Mathis et al. 1977, PAH grains are not considered);

S4: same as $\mathrm{S} 1$, i.e. single-sized dust, with $a_{0}=1 \mu \mathrm{m}$ and $n_{\text {dust }}=$ $3.09 \times 10^{-15} n_{\mathrm{H}_{2}}$

S5: same as S1, i.e. single-sized dust, with $a_{0}=0.02 \mu \mathrm{m}$ and $n_{\text {dust }}=3.87 \times 10^{-10} n_{\mathrm{H}_{2}}$.

The radial profiles of selected chemical species for models $S 1$, $\mathrm{S} 2_{\mathrm{MRN}}$, and $\mathrm{S} 3_{\mathrm{MRN}}$ at time $t_{0}$ are demonstrated in Paper I. The conclusion was that the dust properties, such as size distribution and total number density, have a significant impact on the resulting abundances of all species, especially ions and other charged species.

\subsection{Magnetic diffusivities}

As we mention in the introduction, the chemistry of collapsing clouds has direct consequences on the dynamics via non-ideal MHD effects. We calculate the Ohmic, Hall, and ambipolar diffusivities $\left(\eta_{\mathrm{O}}, \eta_{\mathrm{H}}\right.$, and $\eta_{\mathrm{A}}$, respectively) from the number densities of charged species obtained with our chemistry module (Wardle 2007)

$\eta_{\mathrm{O}}=\frac{c^{2}}{4 \pi \sigma_{\mathrm{O}}}, \eta_{\mathrm{H}}=\frac{c^{2} \sigma_{\mathrm{H}}}{4 \pi \sigma_{\perp}^{2}}$ and $\eta_{\mathrm{A}}=\frac{c^{2} \sigma_{\mathrm{P}}}{4 \pi \sigma_{\perp}^{2}}-\eta_{\mathrm{O}}$,

with $\sigma_{\perp}=\sqrt{\left(\sigma_{\mathrm{H}}^{2}+\sigma_{\mathrm{P}}^{2}\right)}$, where $\sigma_{\mathrm{O}}, \sigma_{\mathrm{H}}, \sigma_{\mathrm{P}}$ are the Ohmic, Hall, and Pedersen conductivities (Cowling 1976; Wardle \& Ng 1999)

$\sigma_{\mathrm{O}}=\frac{e c}{B} \sum_{x} n_{x}\left|q_{x}\right| b_{x}$

$\sigma_{\mathrm{H}}=-\frac{e c}{B} \sum_{x} \frac{n_{x} q_{x} b_{x}^{2}}{1+b_{x}^{2}}$,

$\sigma_{\mathrm{P}}=\frac{e c}{B} \sum_{x} \frac{n_{x}\left|q_{x}\right| b_{x}}{1+b_{x}^{2}}$

where $B$ is the magnetic field, $n_{x}$ and $q_{x}$ are number density and charge of $x$ species. The parameter $b_{x}=\tau_{x} \omega_{x}$ describes how well the charged particles couple to the magnetic field. It is calculated by putting together the cyclotron frequency $\omega_{x}$ and the collisional damping time $\tau_{x}$. A charged species $x$ has cyclotron frequency

$\omega_{x}=\left|q_{x}\right| e B /\left(m_{x} c\right)$,

where $q_{x} e$ is the charge and $m_{x}$ the mass. The damping time of the motion of the charged species relative to the neutrals is

$\tau_{x}=\frac{m_{n}+m_{x}}{m_{n}} \frac{1}{n_{n}\langle\sigma v\rangle_{x n}}$,

where $\langle\sigma v\rangle_{x n}$ is the momentum-transfer rate coefficient for colliding with neutrals, averaged over the Maxwellian velocity distribution. We adopt $\langle\sigma v\rangle_{\mathrm{en}}=10^{-15} \sqrt{128 k_{\mathrm{B}} T /\left(9 \pi m_{\mathrm{e}}\right)}$ for the electrons and $\langle\sigma v\rangle_{\text {in }}=1.9 \times 10^{-9}$ for the ions (Draine et al. 1983). The corresponding rate coefficient for the dust grains follows Eq. (21) in Wardle \& Ng (1999), where the collision speed is the thermal speed of the neutrals since the drift of the grains through the gas is subsonic. For electrons, typically $b_{\mathrm{e}} \gg 1$. The quality of coupling to the magnetic fields drops with the mass of the charged species (for details see Dzyurkevich et al. 2013).

Since our simulations are performed without magnetic fields, we assume that the magnetic pressure scales with gas pressure as $5 B^{2} /(4 \pi)=n k_{\mathrm{B}} T$ (plasma beta $\beta=P_{\text {gas }} / P_{\text {mag }}=5$ ). We then calculate the cyclotronic frequencies and friction times for each charged species, ions, dust, and electrons. Those values are used to determine resistivities (Eqs. (3)-(5)). In the next section, we show the resulting ambipolar, Hall, and Ohmic resistivities, along with radial distribution of charge carriers. We focus only on the comparison of the models with various dust sizes. Changing the initial core radius, i.e. the tempo of collapse, is not affecting the ionisation equilibrium for the chosen parameter range.

\subsection{Fiducial model: evolution of magnetic diffusivities during the collapse}

Figure 1 shows the time evolution of the gas density, temperature, and corresponding magnetic diffusivities for five snapshots, starting from the initial condition and ending with the final output. The fiducial run is stopped when the temperature reaches above $800 \mathrm{~K}$. This is a temperature in which the chemo-erosion of $\mathrm{C}$ from the dust cores has to be included. It can be expected that chemo-erosion greatly affects the chemical abundances because of the large amount of $\mathrm{C}$ released in the gas phase.

The details on the chemical abundances can be found in Paper I. Here we analyse the main trends in the radial behaviour of non-ideal MHD terms. The Ohmic dissipation grows from $10^{12}$ to few of $10^{18} \mathrm{~cm}^{2} \mathrm{~s}^{-1}$ through the simulation. The ambipolar diffusion is shown to dominate other magnetic diffusivities for the most time snapshots of the fiducial simulation, with two exceptions. At $t_{0}-0.221$ we observe that a negative Hall effect is at least as strong as the ambipolar diffusion within $10 \mathrm{AU}$ (i.e. within FHSC radius). Second, at the final output we observe that Ohmic dissipation begins to dominate in the innermost 2-3 AU. The sign reversal of the Hall effect is discussed and explained in the following sections.

\section{Effect of dust properties}

In this section, we analyse how the dust properties affect the charged species and thus the magnetic dissipation. Ohmic, Hall, and ambipolar diffusivities are plotted in Fig. 2, where we compare the outcome of simulations at the moment of FHSC formation. There, the models are arranged according to the mean dust size: model S4 on the left with $\langle a\rangle=1 \mu \mathrm{m}$, followed by fiducial model $\mathrm{S} 1$ and $\mathrm{S} 2_{\mathrm{MRN}}$ with $0.1 \mu \mathrm{m}$ and $0.05 \mu \mathrm{m}$, whereas models $\mathrm{S} 3_{\mathrm{MRN}}$ and $\mathrm{S} 5$ are on the right with mean dust sizes $0.017 \mu \mathrm{m}$ and $0.02 \mu \mathrm{m}$. Even the mean dust size appears to be slightly larger in S5 model; its mean dust cross section is the largest and closest to the value used in Marchand et al. (2016, see also Fig. B.1).

Model S4 with mean dust size of $1 \mu \mathrm{m}$ has the lowest dust number density. The ambipolar diffusion is nevertheless almost as strong as in the fiducial model in the envelope for $r>10^{4} \mathrm{AU}$, where it exceeds $10^{19} \mathrm{~cm}^{2} \mathrm{~s}^{-1}$. Towards the centre of the cloud, the magnetic diffusivities get weaker when compared to the fiducial model: Ohmic resistivity drops to the few of $10^{14} \mathrm{~cm}^{2} \mathrm{~s}^{-1}$ and ambipolar diffusion is about $10^{15} \mathrm{~cm}^{2} \mathrm{~s}^{-1}$ within FHSC. Hall diffusivity dominates within $10 \mathrm{AU}$, and it does not reach above $10^{17} \mathrm{~cm}^{2} \mathrm{~s}^{-1}$ in the centre of the cloud. We can summarise here that in the case in which dust is evolved and can be represented with averaged dust size of $1 \mu \mathrm{m}$, the gas is close to the ideal MHD state and the rotationally supported disk (RSD) cannot be formed.

Model S2 appears to be very close to the fiducial S1 model. The ambipolar diffusion dominates over the whole radial extent, but with its maximum value of $10^{18} \mathrm{~cm}^{2} \mathrm{~s}^{-1}$ it is probably not 

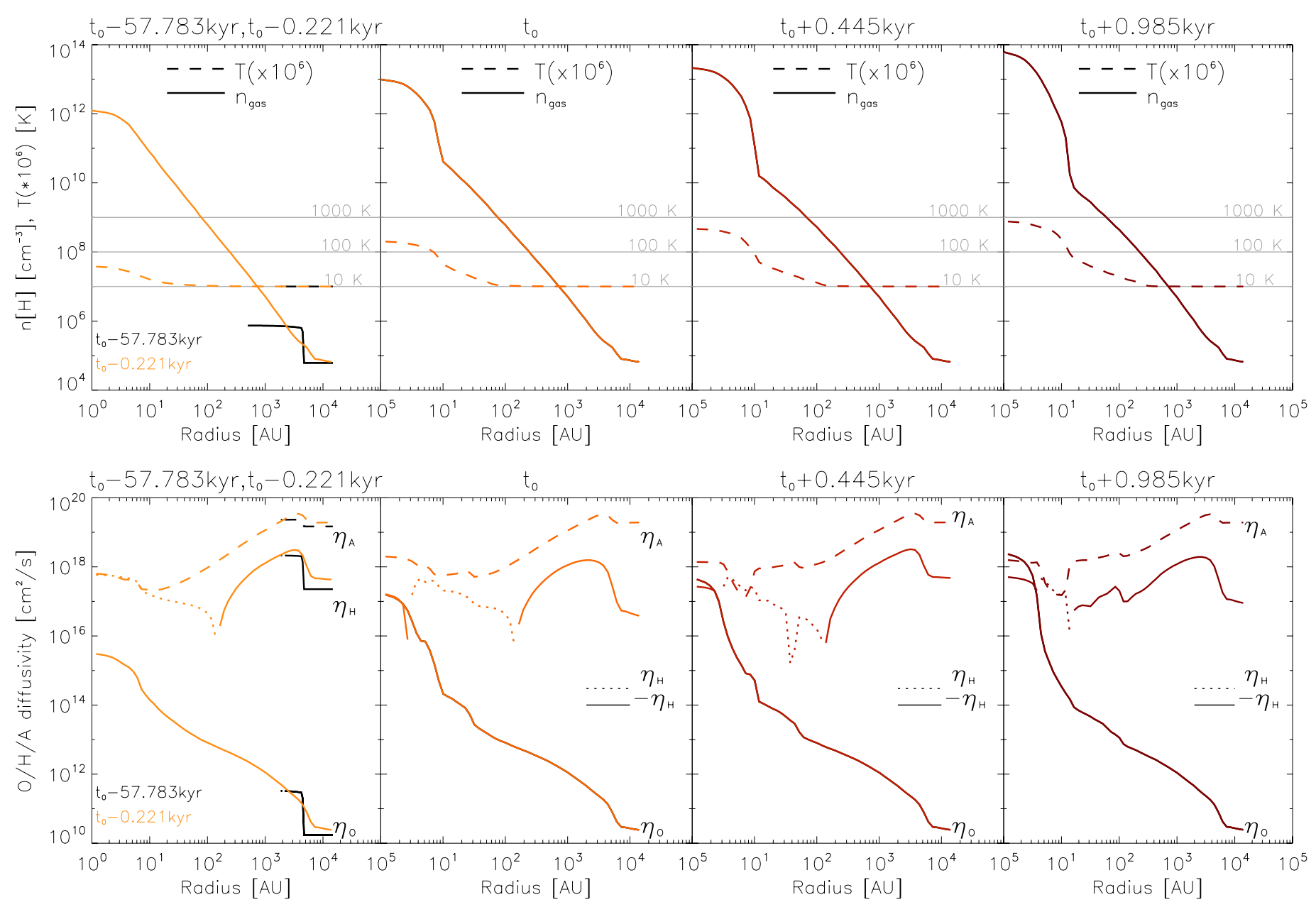

Fig. 1. Time evolution of gas density, temperature, and magnetic diffusivities in the fiducial collapse model, shown for five snapshots. Left plots: both the initial condition (in black) and the data at $t_{0}-0.221 \mathrm{kyr}$, which correspond to the beginning of the adiabatic collapse phase. Right plots: last output, when we stop the simulation.

high enough to enable the formation of RSD around FHSC. The negative Hall effect becomes as strong as the ambipolar diffusion between 4 and $8 \mathrm{AU}$. At the moment of FHSC formation, the Ohmic resistivity is a few orders of magnitude lower in the centre of the cloud.

Models S3 $3_{\text {MRN }}$ and S5 appear to have close values of the mean dust size, but model S5 has a larger dust number density and thus it is placed on the right in Fig. 2. Model S5 has the largest mean grain cross section per hydrogen nucleus, which leads to the largest magnetic diffusivities: Ohmic dissipation exceeds $10^{18} \mathrm{~cm}^{2} \mathrm{~s}^{-1}$ in the very centre of the cloud (see Fig. 2); the Hall effect is negative over the whole radial extend and dominates over ambipolar diffusion between $10 \mathrm{AU}$ and $10^{3} \mathrm{AU}$. Only in $\mathrm{S} 5$ model, the ambipolar diffusion reaches $10^{19} \mathrm{~cm}^{2} \mathrm{~s}^{-1}$ in the cloud centre.

From comparing the magnetic diffusivity profiles in Fig. 2, we can conclude that the chances of forming RSD increase with the increasing mean dust opacity. This means that the small dust have to remain numerous regardless of which size the largest dust grains may achieve. We point out that the Hall effect switches to negative for small dust sizes. In order to understand the impact of the dust properties better, we can ask the following questions: (1) What causes the reversal in the Hall effect? (2) Why does ambipolar diffusion always experience a rise within FHSC, and why does it have a minimum in between $10^{2}$ and $10^{3} \mathrm{AU}$ in case of small dust? and (3) Can we make a simplification in the calculation of the ambipolar diffusion without loss of accuracy, and use a constant ion mass as in Okuzumi (2009) instead of solving a chemical network? We address those questions below.

\subsection{Sign reversal of the Hall effect}

Owing to the total charge neutrality condition $q_{\mathrm{e}} n_{\mathrm{e}}+q_{\mathrm{i}} n_{\mathrm{i}}+$ $q_{\mathrm{G}-} n_{\mathrm{G}-}+q_{\mathrm{G}+} n_{\mathrm{G}+}=0$, where $q_{x}$ is the charge of $x$ species, there are only three independent parameters that can be defined as $n_{\mathrm{e}} / n_{\mathrm{i}}, n_{\mathrm{G}-} / n_{\mathrm{i}}$, and $n_{\mathrm{G}+} / n_{\mathrm{i}}$ (see also Sect. 2 in Xu \& Bai 2016). In Fig. 3 we show the dimensionless parameters $n_{\mathrm{e}} / n_{\mathrm{i}}, n_{\mathrm{G}-} / n_{\mathrm{i}}$, and $n_{\mathrm{G}+} / n_{\mathrm{i}}$. These parameters show how smaller dust size leads to larger inequality $n_{i}>n_{e}$ and the transition to the dust-ion regime and even dust-dust regimes, i.e. when main charge carriers are ions and $\mathrm{G}-\left(\right.$ model $\mathrm{S} 3_{\mathrm{MRN}}$ ), or $\mathrm{G}+$ and $\mathrm{G}-$ (model S5).

The Hall effect term, given in Eq. (2), becomes negative when the Hall conductivity is negative, $\sigma_{\mathrm{H}}<0$. In case of singlecharged dust and local charge neutrality, Eq. (4) can be rewritten as

$\sigma_{\mathrm{H}}=\frac{e c}{B}\left(-\frac{n_{\mathrm{e}}}{1+b_{\mathrm{e}}^{2}}+\frac{n_{\mathrm{i}}}{1+b_{\mathrm{i}}^{2}}+\frac{n_{\mathrm{G}+}-n_{\mathrm{G}-}}{1+b_{\mathrm{G}}^{2}}\right)$,

where we reduce the dust coupling parameter to $b_{\mathrm{G}}$ because it is insensitive to the sign of the charge, i.e. $b_{\mathrm{G}+}=b_{\mathrm{G}-}$. 
N. Dzyurkevich et al.: Magnetic diffusivities in time-dependent chemo-dynamical collapse

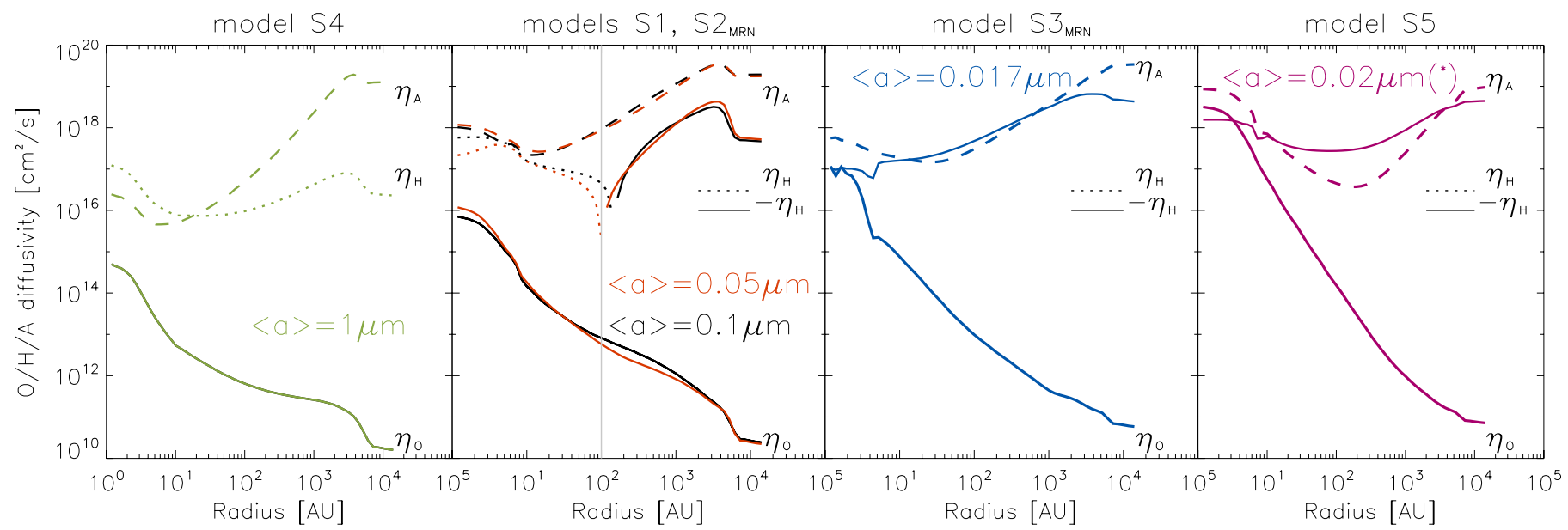

Fig. 2. Magnetic diffusivities for different dust sizes: Ohmic diffusivity (solid line), Hall diffusivity (dotted for positive and dash-dotted for negative), and ambipolar diffusivity (dashed line) vs. radius. The plots show at time $t_{0}-0.22 \mathrm{kyr}$, when central temperature is $T_{c}=55 \mathrm{~K}$ and central density $1.2 \times 10^{12} \mathrm{~cm}^{-3}$. The asterix denotes that the mean dust cross section is minimal in model S5, and the mean dust radius is not a unique characteristic of dust properties.
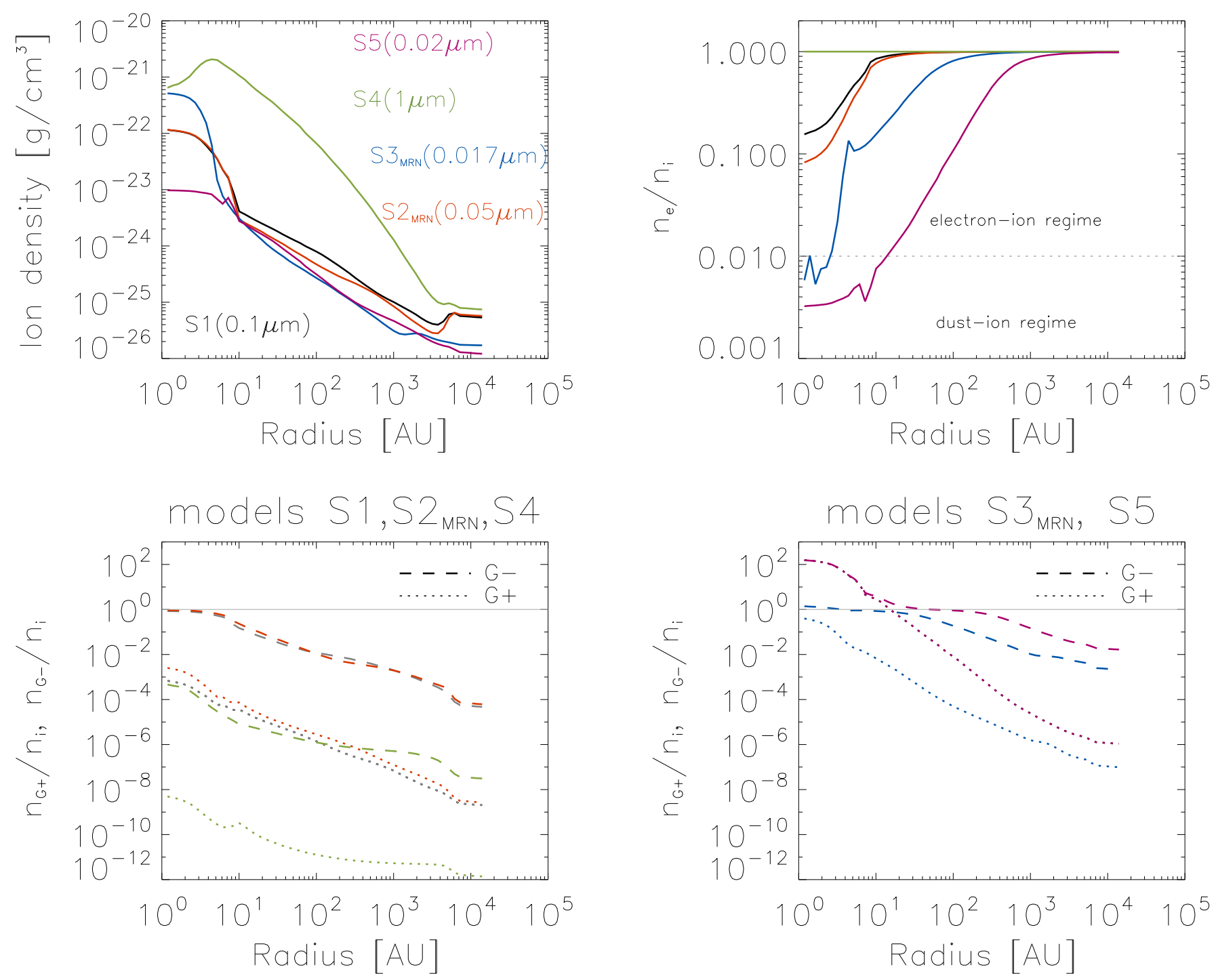

Fig. 3. Various properties of charged species and magnetic diffusivities radial profiles shown for models $\mathrm{S} 1$ (black), $\mathrm{S} 2_{\mathrm{MRN}}$ (red), $\mathrm{S} 3_{\mathrm{MRN}}(\mathrm{blue})$, S4 (green), and S5 (lilac). The colours refer to the same models in all following figures. Plots show, from left to right, the density of ion vs. radius, the relation of electron density to the ion density, and the relative abundances of charged dust to the ion density (bottom). 

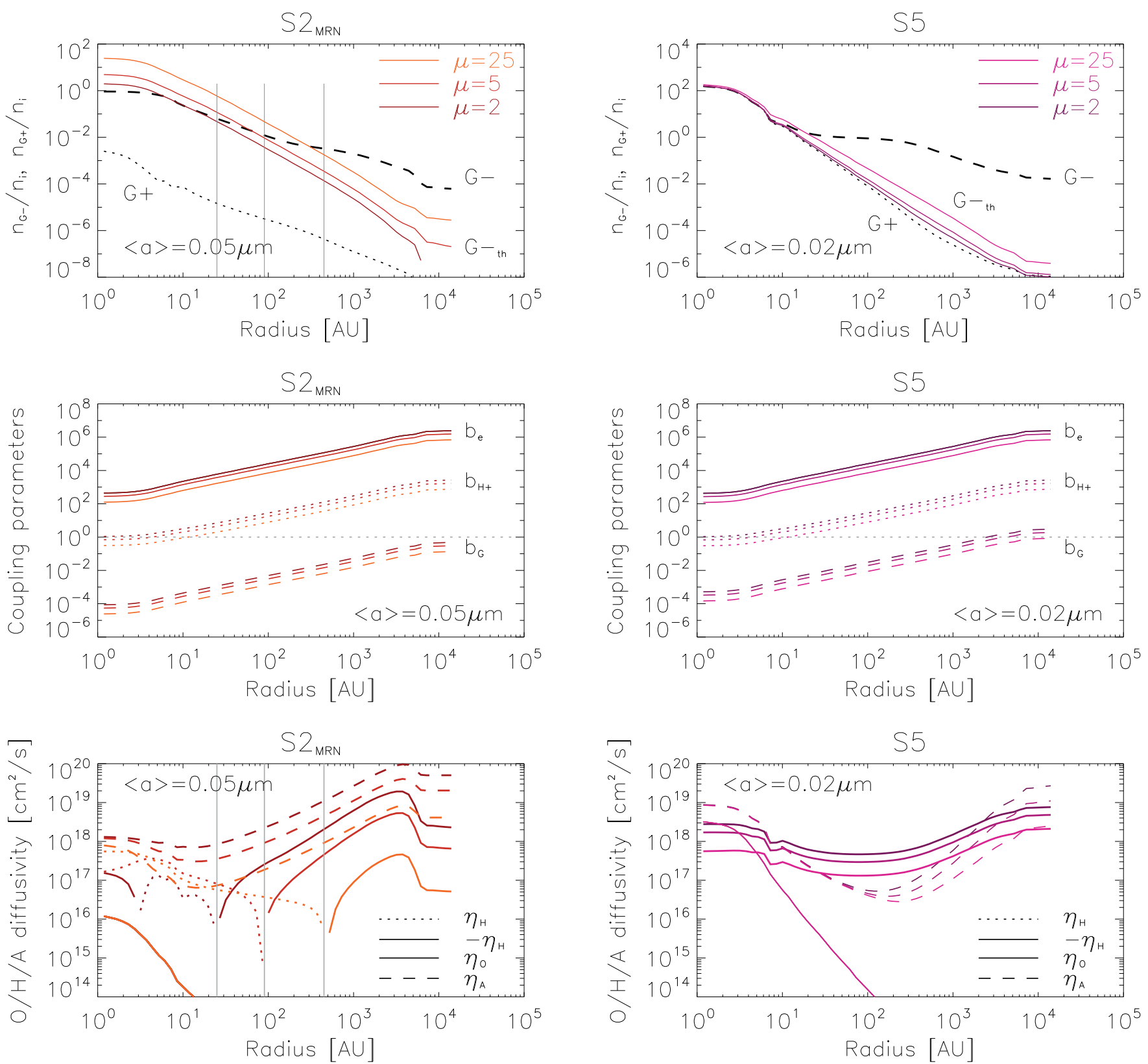

Fig. 4. Top: relative number densities of positively and negatively charged dust, $n_{\mathrm{G}+} / n_{\mathrm{i}}$ and $n_{\mathrm{G}-} / n_{\mathrm{i}}$, compared to the threshold value in Eq. (9). Middle: coupling parameter $b$ for electrons, $\mathrm{H}^{+}$ions, and dust (Eqs. (3)-(5)). Bottom: magnetic diffusivities depending on the magnetic field strength with colours showing $\mu=2,5,25$.

In our simulations, we observe that the coupling parameters $b_{\mathrm{e}} \gg 1$ everywhere, and $b_{\mathrm{i}} \gg 1$ and $b_{\mathrm{G}} \ll 1$ for radii $10 \mathrm{AU}<$ $r<10^{4} \mathrm{AU}$ (see also Fig. 4). Thus, we simplify the equation for $\sigma_{\mathrm{H}}$ further and derive the threshold value for $n_{\mathrm{G}-}^{\text {th }}$,

$n_{\mathrm{G}-}^{\mathrm{th}}=\frac{n_{\mathrm{i}}}{b_{\mathrm{i}}^{2}}-\frac{n_{\mathrm{e}}}{b_{\mathrm{e}}^{2}}+n_{\mathrm{G}+} \cong \frac{n_{\mathrm{i}}}{b_{\mathrm{i}}^{2}}+n_{\mathrm{G}+}$.

When $n_{\mathrm{G}-}>n_{\mathrm{G}-}^{\text {th }}$, the Hall effect changes sign to the negative, which may lead to the interesting MHD effects during the collapse. The Hall effect becomes negative when the number of negative charge carriers, weighted over coupling parameter $b_{x}^{2}$, dominates the number of $b_{x}^{2}$-weighted positive carriers. This is likely to happen when both the negatively charged dust is abundant and the magnetic fields are strong enough for the strong coupling of ions $\left(b_{\mathrm{i}}>1\right)$.
From the previous equations we learn that both the amount of negatively charged dust and also the quality of coupling to the magnetic fields are important. It is now a natural question to ask what happens if we change the cloud magnetisation. We consider cases with $\beta_{\text {plasma }}=4 \pi n k_{\mathrm{B}} T / B^{2}=[2,5,25]$. The various magnetisations are shown with colours in Fig. 4 for models $S 2_{\text {MRN }}$ and S5. We observe that the radial position of the sign reversal in Hall effect shifts from outside to inside, when the magnetic field is increased: $450 \mathrm{AU}$ for $\beta_{\text {plasma }}=25,90 \mathrm{AU}$ for $\beta_{\text {plasma }}=5$ (fiducial case), and $25 \mathrm{AU}$ for $\beta_{\text {plasma }}=2$. At the same time, the Hall effect gets stronger in its absolute value, getting closer to ambipolar dissipation in case of $\mathrm{S} 2_{\mathrm{MRN}}$, or overtaking in case of S5. As we see, the coupling parameter $b_{x}$ changes for different magnetisation, but the common trend of $b_{\mathrm{e}} \gg 1$ and $b_{\mathrm{i}} \gg 1$ and $b_{\mathrm{G}} \ll 1$ is kept for most of radial extend. We find that it is possible to have a reversal in sign of the Hall effect without 
necessary reaching $b_{\mathrm{G}} \cong 1$ as in $\mathrm{Xu} \&$ Bai (2016). We recall here that Xu \& Bai (2016) have been investigating the possibility of Hall effect reversal in the proto-planetary disks, where the coupling parameters for charged species can differ significantly compared to those in the collapsing cloud.

\subsection{Ambipolar diffusion}

The ambipolar diffusion is often implemented in form of $\eta_{\mathrm{A}}=$ $B^{2} /\left(\gamma \rho \rho_{\mathrm{i}}\right)$ in various numerical codes. From this formula, one can see that the ion density is expected to be mainly responsible for the ambipolar diffusion. There are 18 ions included in our network (see Paper I for details). The most massive are $\mathrm{Fe}^{+}$ $\left(55,85 m_{\mathrm{p}}\right)$ and $\mathrm{HCO}^{+}\left(29 m_{\mathrm{p}}\right)$. In the envelope, $\mathrm{CH}_{5}^{+}$is often the most abundant ion, accompanied by $\mathrm{C}^{+}$. From Fig. 5 we can see how the mean ion mass $\left\langle m_{\mathrm{i}}\right\rangle=\rho_{\mathrm{i}} / n_{\mathrm{i}}$ is distributed along the radius. For fiducial case $\mathrm{S} 1$, the mean ion mass is about $17 m_{\mathrm{p}}$ at radii $r>10^{4} \mathrm{AU}$, peaking to $24 m_{\mathrm{p}}$ at $r \simeq 5 \times 10^{3} \mathrm{AU}$, where the density jump from the initial condition was situated. Then the mean ion mass drops to $3 m_{\mathrm{p}}$ when going deeper into the cloud until the border of the FHSC is reached. There, the temperature reaches $55 \mathrm{~K}$ for the snapshot we choose, so the thermal adsorption of most ices is happening and the mean ion mass reaches $24 m_{\mathrm{p}}$ again. There is no clear correlation between the mean ion size and mean dust size, but the trend is similar for all simulations but $\mathrm{S} 4$.

We plot the contribution to Pedersen conductivity for each type of charge carrier in Fig. 6, top. The bottom row in Fig. 6 shows the ambipolar diffusion, calculated in three different ways. The solid line shows ambipolar diffusion, $\eta_{\mathrm{A}}$, according to Eq. (2), using the ion, electron, and charged dust abundances from the chemistry outputs. The dotted line indicates the value of $\eta_{\mathrm{A}}$, where we assume that the number density, $n_{\mathrm{i}}$, is same as in chemical output, but the ion mass is constant everywhere (i.e. $m_{\mathrm{i}}=29 m_{\mathrm{p}}$ to represent $\mathrm{HCO}^{+}$) and not a function of radius as shown in Fig. 5. We plot $\eta_{\mathrm{A}}=B^{2} /\left(\gamma \rho \rho_{\mathrm{i}}\right)$ in Fig. 6 (bottom; dashed line), which would be a correct representation of ambipolar diffusion in the absence of dust, when $n_{\mathrm{e}}=n_{\mathrm{i}}$.

In model S4, the contribution of dust to Pedersen conductivity is negligible and $n_{\mathrm{i}}=n_{\mathrm{e}}$ everywhere (see also Fig. 3). Thus, the dashed and solid lines coincide there. The assumption of a constant ion mass is not too bad either, except that it should be iron with $m_{\mathrm{i}}=55.8 m_{\mathrm{p}}$ instead of $\mathrm{HCO}^{+}$to match the curve more accurately.

For other models, the condition $n_{\mathrm{i}}=n_{\mathrm{e}}$ is not fulfilled and the impact of dust is therefore not negligible. The departure of dashed lines $\left(\eta_{\mathrm{A}}=B^{2} /\left(\gamma \rho \rho_{\mathrm{i}}\right)\right)$ from solid lines (showing $\eta_{\mathrm{A}}$ according to Eq. (2)) start exactly at the radial location where also $n_{\mathrm{i}} \neq n_{\mathrm{e}}$ (see Fig. 3).

We observe that ambipolar diffusion increases for smaller dust steadily from model S4 (left) to model $\mathrm{S} 3_{\mathrm{MRN}}$, but drops for model S5, where the dust is both most abundant and small. This drop occurs when the negatively charged dust is a stronger contributor to the Pedersen conductivity as the ions themself. By comparing with Fig. 3, one can also see that S5 is the only model in which both $\mathrm{G}+$ and $\mathrm{G}-$ are important charge carriers.

To summarise from Fig. 6, we need to solve a chemical network together with collapse to obtain an accurate radial distribution of ion masses. The attempts to simplify using the $\eta_{\mathrm{A}}=$ $B^{2} /\left(\gamma \rho \rho_{\mathrm{i}}\right)$ formulation, or a simpler method to obtain the ionisation of the cloud with fixed ion mass as in Okuzumi (2009), may both lead to one order in magnitude difference in the ambipolar diffusion coefficient.

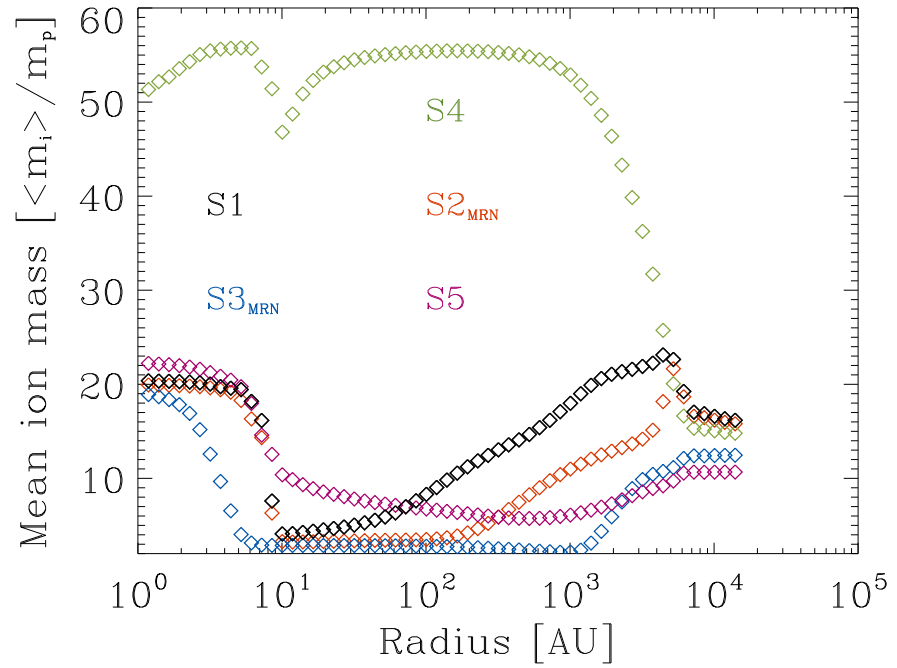

Fig. 5. Snapshot of mean ion mass $m_{\mathrm{i}} / m_{\mathrm{p}}=\rho_{\mathrm{i}} /\left(m_{\mathrm{p}} n_{\mathrm{i}}\right)$ as a function of radius, at time $t_{0}-0.22 \mathrm{kyr}$ (same time snapshot as in Figs. 3-6).

\subsection{Importance of magnetic dissipation mechanisms during the collapse}

For the adopted range in dust size and distribution, we summarise how the dust properties affect the magnetic diffusivities before FHSC formation: (a) Ohmic dissipation increases strongly when the dust size is reduced, but it never dominates; (b) ambipolar diffusion always dominates in the envelope. This property increases as well when the dust size is reduced, but for dust sizes below $0.02 \mu \mathrm{m}$ ambipolar diffusion loses its importance against the Hall effect owing to the charged dust contribution; and (c) the Hall effect gains importance when dust size is reduced, and for dust sizes between 0.1 to $0.05 \mu \mathrm{m}$ it shows the sign reversal. For mean dust sizes smaller than $0.02 \mu \mathrm{m}$, the Hall effect may become negative everywhere.

Recently it has been shown that ambipolar diffusion alone can only lead to small disk sizes in the aligned rotator configuration (Tsukamoto et al. 2015b; Masson et al. 2016). The inclusion of the Hall effect may change the situation dramatically; depending on alignment with magnetic field, large and more massive disks can be formed (Tsukamoto et al. 2015a). Those simulations are still in need of verification, in particular in the context of turbulent dense cores, but these results combined with our findings stress once more the importance of considering the dust properties (size, charge, and growth) of the early stages of stellar formation.

\subsection{Limitations}

There are several limitations in our dynamical chemical solver that can potentially affect the estimate of ionisation and thus the resulting magnetic diffusivities. We discuss below the three main limitations and defer their implementation in our model to future work.

First, we assume that the grain size distribution can be represented by a single mean size. Kunz \& Mouschovias (2009) show that the dust size distribution should be described by at least five bins to have a convergence of less than $1 \%$ in the abundances. More recently, Marchand et al. (2016) find that increasing the number of size bins from one to five can change the non-ideal MHD resistivities up to one order of magnitude, in particular 

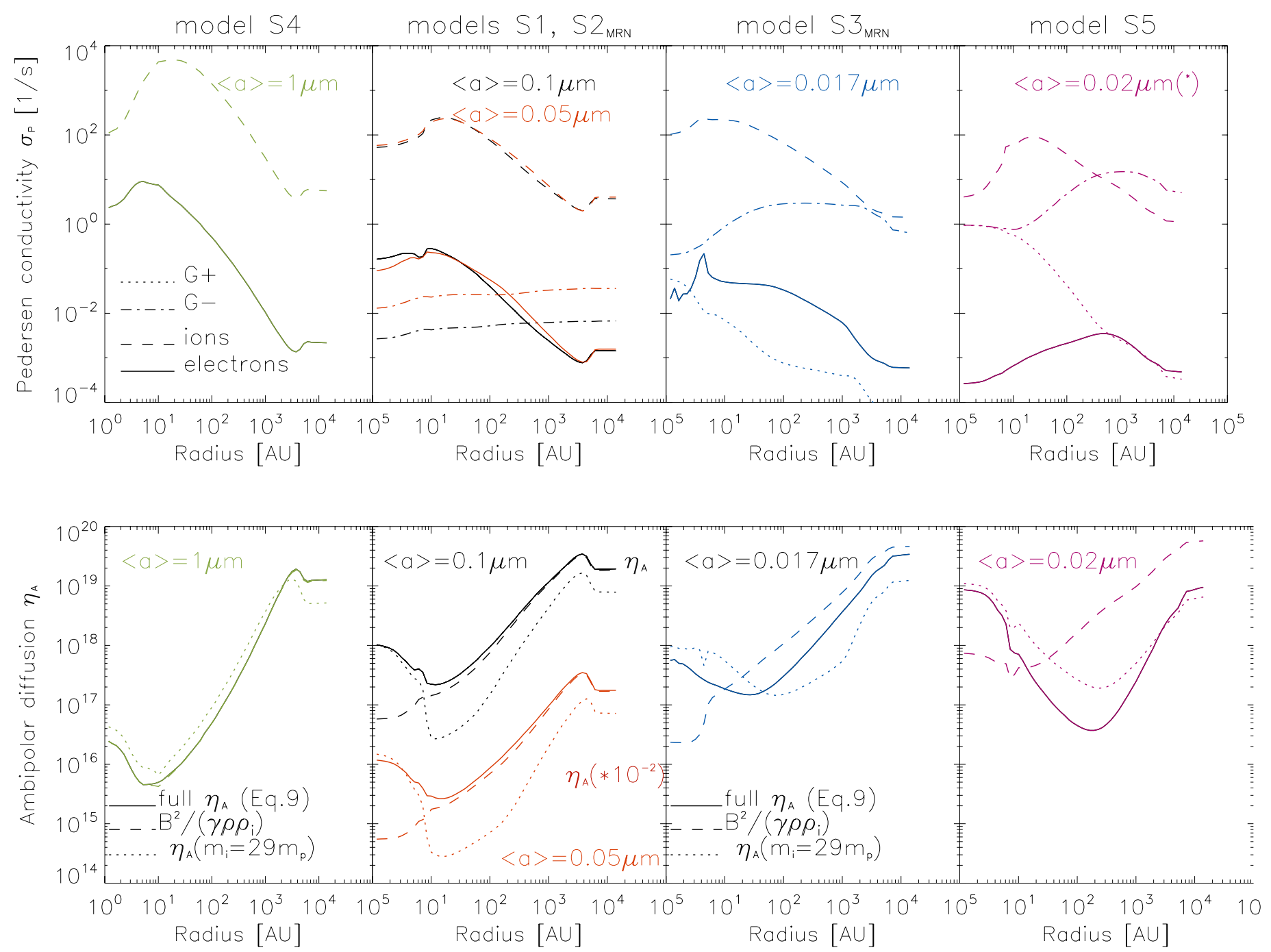

Fig. 6. Top: contribution to Pedersen conductivity for each type of charge carrier. Bottom: ambipolar diffusion $\eta_{\mathrm{A}}$ according to Eq. (2) is shown with solid line, $\eta_{\mathrm{A}}$ under the assumption of overall constant ions mass $m_{\mathrm{i}}=29 m_{\mathrm{p}}$ is shown with dotted line, and a frequently used $\eta_{\mathrm{A}}=B^{2} /\left(\gamma \rho \rho_{\mathrm{i}}\right)$ is shown with dashed line. The asterix in $\langle a\rangle=0.02 \mu \mathrm{m}\left(^{*}\right)$ indicates the mean dust radius is not a unique parameter to characterise the properties of dust and that model S5 has a maximal mean dust cross section.

within the density range where non-ideal effects are expected to play an important role.

Second, we consider single-charged grains whereas they can hold multiple electric charges (Draine \& Sutin 1987; Dzyurkevich et al. 2013). Although multiple charged grains are scarce in dense cores (Umebayashi \& Nakano 1980), they play an important role in the ionisation budget (more multiple charged grains means less single charged grains). Along the same lines, Marchand et al. (2016) show that charge transfer between grains has a first order effect for densities larger than $10^{10}-10^{11} \mathrm{~cm}^{-3}$ and that it becomes even more important in the case of multiple charges. Currently, chemical models are limited by numerical difficulties because of the large dynamical range in the abundance of multiple charged grains, which is hard to handle in classical matrix inversion methods such as DVODE. Analytical models have been developed to treat multiple charges but they lack charge transfer between grains (e.g. Draine \& Sutin 1987) or force the assumption of the constant ion mass (Okuzumi 2009).

Last, we do not account for the variations of the CR ionisation rate deep inside the collapsing core. Padovani et al. (2013, 2014) studied the propagation of CR along magnetic fields lines in collapsing cores, accounting for the effect of CR energy loss and magnetic mirroring. They show how the CR ionisation rate is efficiently reduced in the central region because of the complex magnetic field line configurations found in numerical models of protostellar collapse, making magnetic resistivities larger towards the cloud centre.

\section{Conclusions}

We have performed 3D chemo-dynamical radiationhydrodynamics simulations of $1 M_{\odot}$ isolated dense core collapse. The usage of a chemistry module within the RHD collapse simulations is feasible and beneficial. First, we can now better connect to observations by studying how well the $\mathrm{CO}$ abundance reacts to the parameters and dust of a cloud when computed in chemo-dynamical collapse simulations (see Paper I). Second, the computation of magnetic diffusivities can be performed on the fly in such chemo-dynamical collapse simulations.

We use the chemical abundances resulting from our RHD simulations to study the impact of dust properties on the magnetic dissipation, and interpret the findings in the context of the 
previously performed collapse simulations resulting in the formation of the disks around the protostar. We compare the diffusivities for the chosen range of dust sizes at the moment of FHSC formation, which we define as when cloud centre has $n_{\text {gas }}=1.2 \times 10^{12} \mathrm{~cm}^{-3}$ and $T=55 \mathrm{~K}$. Our main findings are as follows:

- We consider a range in the mean dust sizes, from $1 \mu \mathrm{m}$ to $0.017 \mu \mathrm{m}$, assuming both a single-sized dust and a dustsize distribution. The range of mean dust cross section $\left\langle a^{2}\right\rangle n_{\text {dust }} / n_{\mathrm{H}_{2}}$ is varied from $3.1 \times 10^{-15}$ to $1.4 \times 10^{-13} \mu \mathrm{m}^{2}$. Within this range of dust properties, the gas in the molecular cloud makes a transition from the ionisation state with $n_{\mathrm{e}}=$ $n_{\mathrm{i}}$ in case of $1 \mu \mathrm{m}$ dust, up to the weakly ionised gas where main charge carriers are ions and $G-$, or only charged dust $G-$ and $G+$ in case of $0.02 \mu \mathrm{m}$.

- For the chosen dust size range, the Ohmic diffusivity grows from $7 \times 10^{14} \mathrm{~cm}^{2} \mathrm{~s}^{-1}$ to $4 \times 10^{18} \mathrm{~cm}^{2} \mathrm{~s}^{-1}$ at the cloud centre. Ohmic diffusivity is shown to dominate magnetic dissipation first after the central gas temperature and density and temperature reach $n_{\text {gas }}=10^{14} \mathrm{~cm}^{-3}$ and $T=800 \mathrm{~K}$ (in the fiducial run). For the disk formation it means that Ohmic dissipation alone would lead to the formation of the tiny disk within FHSC.

- The Hall effect increases about one order in magnitude when dust size is reduced from $1 \mu \mathrm{m}$ to $0.017 \mu \mathrm{m}$. We also find that for a mean dust size of $0.1 \mu \mathrm{m}$ the sign of $\eta_{\mathrm{H}}$ becomes negative at $r>90 \mathrm{AU}$, when assuming a moderate cloud magnetisation, $\beta=5$. The strength of the magnetic field affects the radial location of the sign reversal, which is found at $25 \mathrm{AU}$ for $\beta=2$ and at $450 \mathrm{AU}$ for $\beta=25$. We find that the sign reversal occurs when both negatively charged dust dominates and ions are well coupled to the magnetic field, i.e. $n_{\mathrm{G}-}>n_{\mathrm{i}} / b_{\mathrm{i}}^{2}+n_{\mathrm{G}+}$.

- For dust sizes $0.02 \mu \mathrm{m}$ and smaller, the Hall effect is negative overall in the cloud. We expect the effect to become even more pronounced when dust-to-gas ratio is enhanced. The reason is the strong contribution of charged dust to the conductivities, which may even outweigh the contribution of ions.

- We find that ambipolar diffusion always dominates in the envelope in the case of ISM-typical dust sizes and larger up to $1 \mu \mathrm{m}$. It is also reliably dominant for $r>10^{4} \mathrm{AU}$, where it is equal or slightly above $10^{19} \mathrm{~cm}^{2} \mathrm{~s}^{-1}$. Towards the cloud centre, the value of ambipolar diffusion increases from $10^{16} \mathrm{~cm}^{2} \mathrm{~s}^{-1}$ for mean dust size $1 \mu \mathrm{m}$ to $10^{17} \mathrm{~cm}^{2} \mathrm{~s}^{-1}$ for $0.05 \mu \mathrm{m}$, but for smaller dust it declines again and the Hall effect becomes dominant for the large radial range within the envelope.

- We also find that detailed knowledge of ion masses along the radius is important for obtaining the accurate value of ambipolar diffusion.

We conclude that changing the mean dust radius from $1 \mu \mathrm{m}$ to $0.02 \mu \mathrm{m}$ would lead to at least 4 orders in magnitude change for the Ohmic resistivity in the cloud centre to the reversal, up to 2 orders in magnitude increase for the Hall effect, and up to 3 orders in magnitude increase for the ambipolar diffusion in the cloud centre. The Marchand et al. (2016) results are complementary to our study. These results show up to one order in magnitude difference for the magnetic diffusivities caused by accounting for multiple dust charging, the lower value of the CR ionisation rate, and several size bins for dust.

It is interesting to note that we can separate the two scenarios of cloud collapse. In the first scenario, the case in which the average dust size is equal or larger than ISM values is clearly dominated by ambipolar diffusion and results in the formation of relatively small disks according to Hennebelle et al. (2016). The second scenario would be possible when large amounts of much smaller dust, leading to the Hall effect, dominates the collapse; in this case, the results of Tsukamoto et al. (2015a) would apply including factor 10 larger disks when compared with the ambipolar-diffusion-dominated scenario. We propose that the size of circumstellar disks formed in Class 0 objects should correlate with the size of dust and/or the enrichment in dust-to-gas ratio.

Comparing our findings with the previous works (Marchand et al. 2016; Masson et al. 2016; Hincelin et al. 2016) we can conclude that including the dust growth model into the 3D RMHD simulations of the collapse is expected to be vital for our understanding of the non-ideal MHD processes during star formation.

\section{References}

Basu, S., \& Ciolek, G. E. 2004, ApJ, 607, L39

Bellan, P. M. 2008, ApJ, 678, 1099

Braiding, C. R., \& Wardle, M. 2012, MNRAS, 422, 261

Commerçon, B., Hennebelle, P., Audit, E., Chabrier, G., \& Teyssier, R. 2010, A\&A, 510, L3

Commerçon, B., Teyssier, R., Audit, E., Hennebelle, P., \& Chabrier, G. 2011, A\&A, 529, A35

Commerçon, B., Debout, V., \& Teyssier, R. 2014, A\&A, 563, A11

Cowling, T. G. 1976, Magnetohydrodynamics, Monographs on Astronomical Subjects (Bristol: Adam Hilger)

Dapp, W. B., Basu, S., \& Kunz, M. W. 2012, A\&A, 541, A35

Draine, B. T., \& Sutin, B. 1987, ApJ, 320, 803

Draine, B. T., Roberge, W. G., \& Dalgarno, A. 1983, ApJ, 264, 485

Duffin, D. F., \& Pudritz, R. E. 2008, MNRAS, 391, 1659

Duffin, D. F., \& Pudritz, R. E. 2009, ApJ, 706, L46

Dzyurkevich, N., Turner, N. J., Henning, T., \& Kley, W. 2013, ApJ, 765, 114

Dzyurkevich, N., Commerçon, B., Lesaffre, P., \& Semenov, D. 2017, A\&A, submitted [arXiv: 1605.08032]

Flower, D. R., \& Pineau des Forêts, G. 2003, MNRAS, 343, 390

Flower, D. R., \& Pineau des Forêts, G. 2015, A\&A, 578, A63

Fromang, S., Hennebelle, P., \& Teyssier, R. 2006, A\&A, 457, 371

Gerin, M., Pety, J., Fuente, A., et al. 2015, A\&A, 577, L2

Harsono, D., van Dishoeck, E. F., Bruderer, S., Li, Z.-Y., \& Jørgensen, J. K. 2015, A\&A, 577, A22

Hennebelle, P., \& Fromang, S. 2008, A\&A, 477, 9

Hennebelle, P., Commerçon, B., Chabrier, G., \& Marchand, P. 2016, ApJ, 830, L8

Hincelin, U., Wakelam, V., Commerçon, B., Hersant, F., \& Guilloteau, S. 2013, ApJ, 775, 44

Hincelin, U., Commerçon, B., Wakelam, V., et al. 2016, ApJ, 822, 12

Inutsuka, S.-i. 2012, Progr. Theor. Exp. Phys., 2012, 010000

Joos, M., Hennebelle, P., \& Ciardi, A. 2012, A\&A, 543, A128

Joos, M., Hennebelle, P., Ciardi, A., \& Fromang, S. 2013, A\&A, 554, A17

Krasnopolsky, R., \& Königl, A. 2002, ApJ, 580, 987

Krasnopolsky, R., Li, Z.-Y., \& Shang, H. 2010, ApJ, 716, 1541

Kunz, M. W., \& Mouschovias, T. C. 2009, ApJ, 693, 1895

Lazarian, A., Esquivel, A., \& Crutcher, R. 2012, ApJ, 757, 154

Lesaffre, P., Chièze, J.-P., Cabrit, S., \& Pineau des Forêts, G. 2004, A\&A, 427, 147

Lesaffre, P., Belloche, A., Chièze, J.-P., \& André, P. 2005, A\&A, 443, 961

Lesaffre, P., Pineau des Forêts, G., Godard, B., et al. 2013, A\&A, 550, A106

Li, Z.-Y., Krasnopolsky, R., \& Shang, H. 2011, ApJ, 738, 180

Li, Z.-Y., Banerjee, R., Pudritz, R. E., et al. 2014, Protostars and Planets VI, 173

Machida, M. N., Inutsuka, S.-I., \& Matsumoto, T. 2007, ApJ, 670, 1198

Marchand, P., Masson, J., Chabrier, G., et al. 2016, A\&A, 592, 18

Masson, J., Teyssier, R., Mulet-Marquis, C., Hennebelle, P., \& Chabrier, G. 2012, ApJS, 201, 24

Masson, J., Chabrier, G., Hennebelle, P., Vaytet, N., \& Commerçon, B. 2016, A\&A, 587, A32

Mathis, J. S., Rumpl, W., \& Nordsieck, K. H. 1977, ApJ, 217, 425

Mellon, R. R., \& Li, Z.-Y. 2009, ApJ, 698, 922

Mestel, L., \& Spitzer, Jr., L. 1956, MNRAS, 116, 503

Minerbo, G. N. 1978, J. Quant. Spectr. Rad. Transf., 20, 541 
Murillo, N. M., Bruderer, S., van Dishoeck, E. F., et al. 2015, A\&A, 579, A114 Nakano, T., Nishi, R., \& Umebayashi, T. 2002, ApJ, 573, 199

Okuzumi, S. 2009, ApJ, 698, 1122

Padovani, M., Hennebelle, P., \& Galli, D. 2013, A\&A, 560, A114

Padovani, M., Galli, D., Hennebelle, P., Commerçon, B., \& Joos, M. 2014, A\&A 571, A33

Pagani, L., Lefèvre, C., Bacmann, A., \& Steinacker, J. 2012, A\&A, 541, A154

Pérez, L. M., Carpenter, J. M., Andrews, S. M., et al. 2016, Science, 353, 1519

Seifried, D., Banerjee, R., Pudritz, R. E., \& Klessen, R. S. 2015, MNRAS, 446, 2776

Semenov, D., Henning, T., Helling, C., Ilgner, M., \& Sedlmayr, E. 2003, A\&A, 410,611

Steinacker, J., Pagani, L., Bacmann, A., \& Guieu, S. 2010, A\&A, 511, A9

Tassis, K., Hezareh, T., \& Willacy, K. 2012a, ApJ, 760, 57

Tassis, K., Willacy, K., Yorke, H. W., \& Turner, N. J. 2012b, ApJ, 753, 29

Tassis, K., Willacy, K., Yorke, H. W., \& Turner, N. J. 2012c, ApJ, 754, 6

Teyssier, R. 2002, A\&A, 385, 337

Teyssier, R., Fromang, S., \& Dormy, E. 2006, J. Comput. Phys., 218, 44

Tobin, J. J., Looney, L. W., Wilner, D. J., et al. 2015, ApJ, 805, 125

Tobin, J. J., Kratter, K. M., Persson, M. V., et al. 2016a, Nature, 538, 483

Tobin, J. J., Looney, L. W., Li, Z.-Y., et al. 2016b, EAS Pub. Ser., 75, 273

Tomida, K., Okuzumi, S., \& Machida, M. N. 2015, ApJ, 801, 117

Tsukamoto, Y., Iwasaki, K., Okuzumi, S., Machida, M. N., \& Inutsuka, S. 2015a, ApJ, 810, L26

Tsukamoto, Y., Iwasaki, K., Okuzumi, S., Machida, M. N., \& Inutsuka, S. 2015b, MNRAS, 452, 278

Umebayashi, T., \& Nakano, T. 1980, PASJ, 32, 405

Wardle, M. 2007, Ap\&SS, 311, 35

Wardle, M., \& Ng, C. 1999, MNRAS, 303, 239

Wolfire, M. G., Hollenbach, D., McKee, C. F., Tielens, A. G. G. M., \& Bakes, E. L. O. 1995 , ApJ, 443,152

Wurster, J., Price, D. J., \& Bate, M. R. 2016, MNRAS, 457, 1037

Xu, R., \& Bai, X.-N. 2016, ApJ, 819, 68

\section{Appendix A: Approximate treatment of the dust-size distribution}

In our chemical module, we want to take into account the effect of dust-size distribution on the chemistry and to avoid high memory costs due to treatment of numerous size bins. Here, we trace the number densities only of species G0, G-, and G+. For simplicity, we neglect the multiple dust charges. In case of fixed dust size, the density of dust grain is simply defined as

$n_{\text {dust }}=\frac{M_{\text {gas }} f_{\text {dg }}}{4 / 3 \pi a^{3} \rho_{\text {solid }}}$,

where $a$ is the grain radius and $\rho_{\text {solid }}$ is the internal density of the solid materials. We adopt a MRN size distribution $\mathrm{d} n / \mathrm{d} a \propto a^{-3.5}$ (Mathis et al. 1977).

Next, the dust radius can be fixed, as in models S1, S4, and $\mathrm{S} 5$, or one can choose a range of sizes: the minimum grain radius is $a_{\mathrm{min}}=0.01 \mu \mathrm{m}$ and maximum radius $a_{\mathrm{max}}=0.3 \mu \mathrm{m}$ (models $\mathrm{S} 2_{\mathrm{MRN}}$ and $\mathrm{S} 3_{\mathrm{MRN}}$ ). For the chemistry, the rates of ion-dust or electron dust reactions are directly affected by number density of the dust. The total available surface of dust grains affects the rates of the freeze out of species on dust. We can determine $n_{\text {dust }}$ using Eq. (A.1) after calculating the effective cubic radius $\left\langle a^{3}\right\rangle$

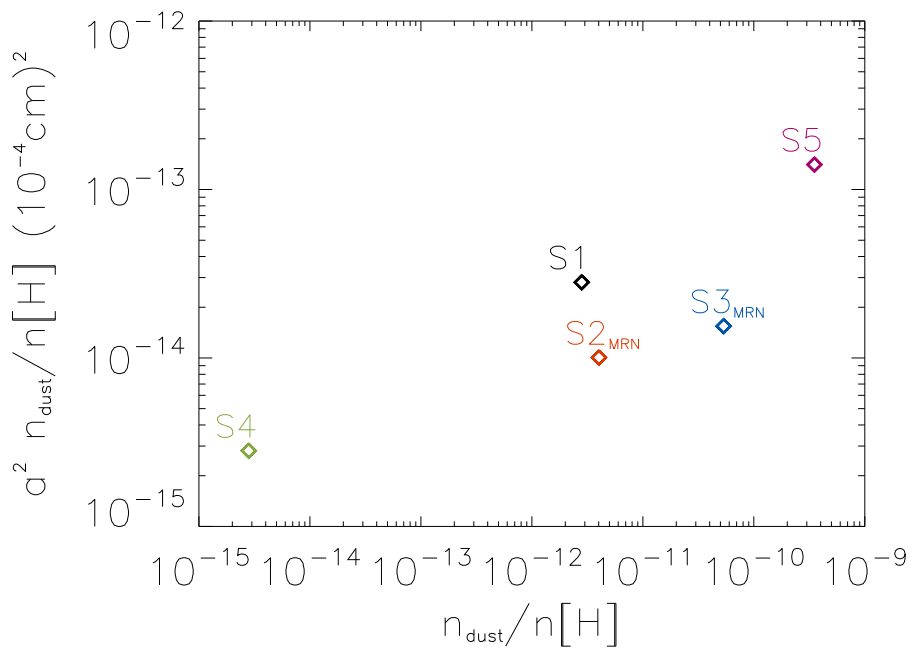

Fig. B.1. Mean grain cross section per hydrogen nucleus as a function of dust density $n_{\text {dust }}$ for models $\mathrm{S} 1, \mathrm{~S} 4, \mathrm{~S} 5, \mathrm{~S} 2_{\mathrm{MRN}}$, and $\mathrm{S} 3_{\mathrm{MRN}}$. Model $\mathrm{S} 2_{\mathrm{MRN}}$ is taken closest to the coreshine modelling.

from

$\left\langle a^{3}\right\rangle=\frac{\int_{a_{\min }}^{a_{\max }} a^{3} a^{-3.5} \mathrm{~d} a}{\int_{\min }^{\max } a^{-3.5} \mathrm{~d} a}=5 \frac{a_{\mathrm{max}}^{0.5}-a_{\min }^{0.5}}{a_{\mathrm{min}}^{-2.5}-a_{\mathrm{max}}^{-2.5}}$.

Similarly, we can use the number-weighted squared radius $\left\langle a^{2}\right\rangle$ for reactions sensitive to dust surface, and define the dust mean opacity (see Appendix B).

$\left\langle a^{2}\right\rangle=2.5 \frac{a_{\mathrm{min}}^{-0.5}-a_{\mathrm{max}}^{-0.5}}{a_{\mathrm{min}}^{-2.5}-a_{\mathrm{max}}^{-2.5}}$.

In Table 1, the average grain radius is

$\langle a\rangle=2.5 \frac{a_{\mathrm{min}}^{-1.5}-a_{\mathrm{max}}^{-1.5}}{a_{\mathrm{min}}^{-2.5}-a_{\max }^{-2.5}} / 1.5$.

\section{Appendix B: Mean dust opacity or cross section}

We would like to emphasise here the importance of dust mean opacity $\left\langle a^{2}\right\rangle n_{\text {dust }}$, as a value that also affects the ionisation state of the gas strongly. The creation rate in case of the charge transfer or the recombination is proportional to $n_{\text {dust }}$. The creation rate for ice species is proportional to $\left\langle a^{2}\right\rangle n_{\text {dust }}$, whereas the dust density decreases steadily from model $\mathrm{S} 1$ to $\mathrm{S} 3_{\mathrm{MRN}}$, the parameter $\left\langle a^{2}\right\rangle n_{\text {dust }}$ is maximal for $\mathrm{S} 1$, followed by $\mathrm{S} 3_{\mathrm{MRN}}$, with a minimum value in $\mathrm{S} 2_{\mathrm{MRN}}$ (see Fig. B.1). Cases S4 and S5 represent low dust impact and high dust impact cases, correspondingly. 